\title{
Association of Antimicrobial Resistance in Campylobacter spp. in Broilers and Turkeys with Antimicrobial Use
}

\author{
Bernd-Alois Tenhagen ${ }^{1, *}$, Matthias Flor ${ }^{1} \oplus$, Katja Alt ${ }^{1}$, Marie-Theres Knüver ${ }^{2}$, Christiane Buhler ${ }^{2}$, \\ Annemarie Käsbohrer ${ }^{1}$ (D) and Kerstin Stingl ${ }^{2}$ \\ 1 Unit Epidemiology, Zoonoses and Antimicrobial Resistance, Department Biological Safety, German Federal \\ Institute for Risk Assessment, Max-Dohrn-Str. 8-10, 10589 Berlin, Germany; Matthias.Flor@bfr.bund.de (M.F.); \\ Katja.Alt@bfr.bund.de (K.A.); Annemarie.kaesbohrer@bfr.bund.de (A.K.) \\ 2 Unit Food Microbiology, Pathogen-Host Interactions, Department Biological Safety, German Federal Institute \\ for Risk Assessment, Max-Dohrn-Str. 8-10, 10589 Berlin, Germany; \\ Marie-Theres.knuever@bfr.bund.de (M.-T.K.); Christiane.Buhler@bfr.bund.de (C.B.); \\ Kerstin.Stingl@bfr.bund.de (K.S.) \\ * Correspondence: Bernd-Alois.Tenhagen@bfr.bund.de; Tel.: +49-301841224301
}

\section{check for} updates

Citation: Tenhagen, B.-A.; Flor, M.; Alt, K.; Knüver, M.-T.; Buhler, C.; Käsbohrer, A.; Stingl, K. Association of Antimicrobial Resistance in Campylobacter spp. in Broilers and Turkeys with Antimicrobial Use. Antibiotics 2021, 10, 673. https:// doi.org/10.3390/antibiotics10060673

Academic Editors: Magdalena

Rzewuska, Lucjan Witkowski and Marina Spînu

Received: 14 April 2021

Accepted: 29 May 2021

Published: 4 June 2021

Publisher's Note: MDPI stays neutral with regard to jurisdictional claims in published maps and institutional affiliations.

Copyright: (c) 2021 by the authors. Licensee MDPI, Basel, Switzerland. This article is an open access article distributed under the terms and conditions of the Creative Commons Attribution (CC BY) license (https:/ / creativecommons.org/licenses/by/ $4.0 /)$.

\begin{abstract}
We investigated trends in antimicrobial resistance (AMR) in Campylobacter jejuni and Campylobacter coli in poultry between 2010 and 2016 in Germany and their association with antimicrobial use. Campylobacter had been isolated from the caeca of broilers and turkeys at slaughter by regional laboratories according to current ISO methods in the framework of a national monitoring program. Isolates were submitted to the National Reference Laboratory for Campylobacter and tested for AMR using broth microdilution methods. Minimum inhibitory concentrations were evaluated according to epidemiological cut-off values. Antimicrobial use (AMU) data from 2014 to 2016 were taken from a government report. AMR was higher in C. coli than in C. jejuni and higher in turkeys than in broilers. AMR was highest to tetracycline and the tested (fluoro)quinolones while it was rare to gentamicin in both bacterial species, infrequent to erythromycin in C. jejuni, and moderate in C. coli. AMR to tetracycline and erythromycin decreased over time while it increased to (fluoro)quinolones. An association of AMU and AMR was observed for resistance to tetracycline and erythromycin, while it was not observed for the aminoglycosides. Resistance to nalidixic acid and ciprofloxacin increased despite a decrease of fluoroquinolone use between 2014 and 2016, indicating that other factors have a strong influence on resistance to (fluoro)quinolones in Campylobacter.
\end{abstract}

Keywords: Campylobacter; antimicrobial resistance; antimicrobial use; poultry

\section{Introduction}

Campylobacteriosis is the most frequent foodborne enteric disease in humans in Germany and the European Union (EU) [1]. Most Campylobacter infections are self-limiting but severe and systemic infections may require antimicrobial treatment. A recent study observed that in around one third of the cases, campylobacteriosis was treated with antimicrobials in Germany [2]. Therefore, from a public health perspective, limiting antimicrobial resistance (AMR) in Campylobacter spp. is of utmost importance. As most Campylobacter infections in humans are foodborne, AMR in Campylobacter from animals and food has a substantial impact on AMR in Campylobacter causing infections in humans [3]. This is especially valid for Campylobacter from poultry, as several studies have attributed poultry a major role as a source of human infections $[2,4]$.

In recent years, AMR in Campylobacter from poultry in Europe was monitored based on Commission Implementing Decision 2013/652/EU [5]. In that framework, AMR investigation in C. jejuni from broilers and turkeys was mandatory every two years in Germany. To this end, caecal samples from broilers and turkeys were collected at slaughter and tested for thermophilic Campylobacter spp. Sampling plans had to be designed to collect a minimum 
of 170 isolates of $C$. jejuni from each of these poultry populations [5]. In a number of countries, an increase of resistance of $C$. jejuni from broilers to tetracycline and ciprofloxacin was observed. This was less frequent in isolates from turkeys; however, monitoring of AMR is not mandatory in all countries. Likewise, $C$. jejuni isolates from humans showed increasing resistance to ciprofloxacin and tetracyclin in a number of countries [6]. In Germany, active monitoring of AMR in Campylobacter spp. from poultry had already been carried out since 2010, albeit at other intervals. The EU-harmonized protocol is supplemented by additional testing of $C$. coli collected within the same sampling framework.

Antimicrobial use (AMU) is known to be one of the main drivers for AMR in bacteria. As the prevalence of Campylobacter spp. in the German broiler population is typically high and Campylobacter spp. is spread among flocks and their environments, any AMU in poultry can be expected to exert selective pressure, favoring the colonization of resistant Campylobacter spp. in these poultry populations. Vice versa, a reduction of AMU in poultry may lead to a subsequent reduction of AMR in Campylobacter from these populations. Such an association of use and AMR has been shown for E. coli in several studies and animal species [7-9].

For Campylobacter, however, the situation seems to be more complex. A joint report by the European Food Safety Authority (EFSA), the European Center for Disease Control and Prevention (ECDC), and the European Medicines Agency (EMA) on the association of AMU and AMR in animals and humans has pointed out that on a national scale, use of fluoroquinolones in poultry and resistance to the fluoroquinolone ciprofloxacin in C. jejuni from poultry were positively associated [3]. However, analyses comparing resistance prevalence in isolates from conventional and organic turkey meat found significant differences for $C$. jejuni, but not for C. coli [10]. Likewise, a recent study demonstrated differences in AMR of Campylobacter spp. originating from organic and conventional pig production for France, but not for Sweden [11]. In addition, a study from the UK found that the resistance level of Campylobacter spp. to fluoroquinolones was influenced by AMU on the poultry farm, but that it was also present on many farms that never used fluoroquinolones [8].

It was the objective of this study to investigate whether changes in the use of antimicrobials in broilers and turkeys in the course of the "German antimicrobial minimization strategy" were associated with changes in resistance of $C$. jejuni and C. coli from these poultry species. Our hypothesis was that use of antimicrobials would be positively associated with resistance, i.e., increases and decreases in use would be associated with increases and decreases in resistance, respectively. For the years 2010 to 2013, no species-specific AMU data were available, but the overall decrease in antimicrobial sales to veterinarians indicated decreasing use [12]. For the period from the second half of 2014 to 2016, we could use species and antimicrobial class-specific use data to test our hypothesis.

\section{Results}

\subsection{Development of AMU in Broilers and Turkeys}

Among the considered substances, AMU in broilers was highest for aminoglycosides, followed by fluoroquinolones (Table 1). Compared to the first observation period (i.e., second half of 2014), AMU was lower for all recorded substances in 2016 than in 2014. Total AMU in broilers decreased from a treatment frequency (TF) of 26.0 in the second half of 2014 to a TF of 17.4 in $2016(-33.0 \%)$. TF is the number of days an average animal is under treatment with an antibiotic in a six month period (for details see material and methods). The biggest decrease among the specifically included substance classes was observed for macrolides $(-62 \%)$, and tetracyclines $(-58 \%)$. The smallest decrease was observed for aminoglycosides $(-27 \%)$ and fluoroquinolones $(-15 \%)$. 
Table 1. Antimicrobial use by antimicrobial class in broilers and turkeys in Germany from the second half of 2014 to the second half of 2016, measured as treatment frequency (TF). Data from [13].

\begin{tabular}{|c|c|c|c|c|c|}
\hline Species/6 Month Period & Aminoglycosides & Fluoroquinolones & Macrolides & Tetracyclines & Total \\
\hline \multicolumn{6}{|l|}{ Broilers } \\
\hline 2014/2 (July-December) & 5.83 & 1.76 & 1.05 & 0.41 & 25.97 \\
\hline 2015/1 (January-June) & 4.40 & 1.88 & 0.97 & 0.18 & 20.42 \\
\hline 2015/2 (July-December) & 3.28 & 1.80 & 0.63 & 0.15 & 16.15 \\
\hline 2016/1 (January-June) & 3.43 & 1.61 & 0.49 & 0.24 & 16.57 \\
\hline 2016/2 (July-December) & 5.13 & 1.38 & 0.30 & 0.11 & 18.25 \\
\hline 2016 combined $^{1}$ & 4.28 & 1.50 & 0.39 & 0.17 & 17.41 \\
\hline$\%$ change $2014-2016^{2}$ & -26.6 & -14.7 & -62.4 & -57.5 & -33.0 \\
\hline \multicolumn{6}{|l|}{ Turkeys } \\
\hline 2014/2 (July-December) & 0.59 & 3.47 & 2.08 & 2.27 & 33.01 \\
\hline 2015/1 (January-June) & 0.72 & 3.48 & 1.68 & 1.85 & 29.21 \\
\hline 2015/2 (July-December) & 0.50 & 2.76 & 1.57 & 1.98 & 25.73 \\
\hline 2016/1 (January-June) & 0.57 & 2.92 & 1.27 & 1.85 & 24.40 \\
\hline 2016/2 (July-December) & 0.65 & 2.19 & 1.29 & 1.39 & 22.37 \\
\hline 2016 combined $^{1}$ & 0.61 & 2.55 & 0.50 & 1.28 & 23.39 \\
\hline$\%$ change $2014-2016^{2}$ & +3.6 & -26.5 & -38.1 & -38.3 & -29.2 \\
\hline
\end{tabular}

${ }^{1}$ mean of $2016 / 1$ and $2016 / 2{ }^{2} \%$ change from $2014 / 2$ to 2016 combined.

Among the considered substance groups, the most frequently used drug class in turkeys was the fluoroquinolones, followed by tetracyclines and macrolides. Aminoglycosides were less frequently used in turkeys than the other substances (Table 1).

In turkeys, total use decreased from a TF of 33.0 in 2014 to 23.4 in 2016 (-29\%). Among the frequently used substance classes with relevance to Campylobacter, the reduction was biggest for macrolides $(-38 \%)$, followed by tetracyclines $(-28 \%)$ and fluoroquinolones $(-26 \%)$. In contrast, use of aminoglycosides increased slightly in the observation period $(+4 \%)$. However, in contrast to the situation in broilers, use of aminoglycosides was low in turkeys during the whole observation period (Table 1).

\subsection{Development of $A M R$}

A total of 1792 isolates were tested for their resistance to the six antimicrobials. Of those, 659 were from broilers (191 C. coli, 468 C. jejuni) and 1133 from turkeys (587 C. coli, 546 C. jejuni). Results from resistance testing are shown in Table 2. Resistance in both animal and bacterial species was highest to the (fluoro)quinolones ciprofloxacin and nalidixic acid as well as to tetracycline. For gentamicin, there were only two resistant isolates, one C. jejuni from broilers in 2016 and one C. coli from turkeys in 2014. Therefore, gentamicin was not included in the logistic regression models.

Full susceptibility was more frequent in C. jejuni than in C. coli, and it was more likely to be observed in isolates from broilers than from turkeys in both models (Tables 3 and 4). The proportion of fully susceptible isolates did not change significantly over the years in broilers and turkeys (Table 3). 
Table 2. Proportion of resistance (\% and $95 \%$ confidence interval [14]) in C. jejuni and C. coli from broilers at slaughter in Germany to six antimicrobials.

\begin{tabular}{|c|c|c|c|c|c|c|c|}
\hline $\begin{array}{c}\text { Species, Origin } \\
\text { Year } \\
\text { No. of Isolates }\end{array}$ & Nalidixic Acid & Ciprofloxacin & Gentamicin & Streptomycin & Tetracycline & Erythromycin & Fully Susceptible \\
\hline \multicolumn{8}{|c|}{ C. coli, Broiler } \\
\hline $2011, N=25$ & $\begin{array}{c}88.0 \\
(69.2-96.7)\end{array}$ & $\begin{array}{c}92.0 \\
(73.9-98.9)\end{array}$ & $\begin{array}{c}0 \\
(0-23.1)\end{array}$ & $\begin{array}{c}12.0 \\
(3.5-31.0)\end{array}$ & $\begin{array}{c}80.0 \\
(60.3-91.4)\end{array}$ & $\begin{array}{c}32.0 \\
(17.2-51.8)\end{array}$ & $\begin{array}{c}0 \\
(0-16.1)\end{array}$ \\
\hline $2013, N=16$ & $\begin{array}{c}81.3 \\
(56.2-94.2)\end{array}$ & $\begin{array}{c}81.3 \\
(56.2-94.2)\end{array}$ & $\begin{array}{c}0 \\
(0-4.1)\end{array}$ & $\begin{array}{c}6.3 \\
(0-30.6)\end{array}$ & $\begin{array}{c}68.8 \\
(44.1-85.9)\end{array}$ & $\begin{array}{c}0 \\
(0-23.1)\end{array}$ & $\begin{array}{c}12.5 \\
(2.5-37.5)\end{array}$ \\
\hline $2014, \mathrm{~N}=112$ & $\begin{array}{c}79.5 \\
(71.0-86.0)\end{array}$ & $\begin{array}{c}82.1 \\
(73.9-88.2)\end{array}$ & $\begin{array}{c}0 \\
(0-11.2)\end{array}$ & $\begin{array}{c}12.5 \\
(7.5-20.1)\end{array}$ & $\begin{array}{c}82.1 \\
(73.9-88.2)\end{array}$ & $\begin{array}{c}23.2 \\
(16.4-31.9)\end{array}$ & $\begin{array}{c}6.3 \\
(2.9-12.6)\end{array}$ \\
\hline $2016, N=38$ & $\begin{array}{c}84.2 \\
(69.2-92.9) \\
\end{array}$ & $\begin{array}{c}92.1 \\
(78.5-98.0) \\
\end{array}$ & $\begin{array}{c}0 \\
(0-2.4) \\
\end{array}$ & $\begin{array}{c}18.4 \\
(9.0-33.8)\end{array}$ & $\begin{array}{c}86.8 \\
(72.1-94.6) \\
\end{array}$ & $\begin{array}{c}5.3 \\
(0.6-18.4)\end{array}$ & $\begin{array}{c}2.6 \\
(0-14.9)\end{array}$ \\
\hline \multicolumn{8}{|c|}{ C. coli, Turkey } \\
\hline $2010, N=76$ & $\begin{array}{c}92.1 \\
(83.5-96.6)\end{array}$ & $\begin{array}{c}93.4 \\
(85.2-97.5)\end{array}$ & $\begin{array}{c}0 \\
(0-5.7)\end{array}$ & $\begin{array}{c}13.2 \\
(7.2-22.8)\end{array}$ & $\begin{array}{c}92.1 \\
(83.4-96.6)\end{array}$ & $\begin{array}{c}64.5 \\
(53.2-74.3)\end{array}$ & $\begin{array}{c}2.6 \\
(0.2-9.8)\end{array}$ \\
\hline $2012, N=79$ & $\begin{array}{c}87.3 \\
(78.1-93.2)\end{array}$ & $\begin{array}{c}92.4 \\
(84.1-96.8)\end{array}$ & $\begin{array}{c}0.4 \\
(0-2.4)\end{array}$ & $\begin{array}{c}16.5 \\
(9.8-26.4)\end{array}$ & $\begin{array}{c}93.7 \\
(85.6-97.5)\end{array}$ & $\begin{array}{c}35.4 \\
(25.8-46.5)\end{array}$ & $\begin{array}{c}3.8 \\
(0.9-11.1)\end{array}$ \\
\hline $2014, N=263$ & $\begin{array}{c}87.8 \\
(83.3-91.3)\end{array}$ & $\begin{array}{c}91.6 \\
(87.6-94.5)\end{array}$ & $\begin{array}{c}0 \\
(0-2.7)\end{array}$ & $\begin{array}{c}14.1 \\
(10.4-18.8)\end{array}$ & $\begin{array}{c}88.2 \\
(83.7-91.6)\end{array}$ & $\begin{array}{c}35.0 \\
(29.5-40.9)\end{array}$ & $\begin{array}{c}2.7 \\
(1.2-5.5)\end{array}$ \\
\hline \multicolumn{8}{|c|}{ C. jejuni, Broiler } \\
\hline $2011, N=60$ & $\begin{array}{c}58.3 \\
(45.7-69.9)\end{array}$ & $\begin{array}{c}65.0 \\
(52.3-75.9)\end{array}$ & $\begin{array}{c}0 \\
(0-10.4)\end{array}$ & $\begin{array}{c}5.0 \\
(1.2-14.3)\end{array}$ & $\begin{array}{c}50.0 \\
(37.7-62.3)\end{array}$ & $\begin{array}{c}3.3 \\
(0.3-12.0)\end{array}$ & $\begin{array}{c}18.3 \\
(10.5-30.2)\end{array}$ \\
\hline $2013, N=40$ & $\begin{array}{c}42.5 \\
(28.5-57.8)\end{array}$ & $\begin{array}{c}47.5 \\
(32.9-62.5)\end{array}$ & $\begin{array}{c}0 \\
(0-2.2)\end{array}$ & $\begin{array}{c}2.5 \\
(0-14.0)\end{array}$ & $\begin{array}{c}32.5 \\
(20.0-48.1)\end{array}$ & $\begin{array}{c}0 \\
(0-10.4)\end{array}$ & $\begin{array}{c}42.5 \\
(28.5-57.8)\end{array}$ \\
\hline $2014, N=202$ & $\begin{array}{c}55.0 \\
(48.1-61.7)\end{array}$ & $\begin{array}{c}65.8 \\
(59.1-72.0)\end{array}$ & $\begin{array}{c}0.6 \\
(0-3.7)\end{array}$ & $\begin{array}{c}0 \\
(0-2.2)\end{array}$ & $\begin{array}{c}50.5 \\
(43.7-57.3)\end{array}$ & $\begin{array}{c}3.5 \\
(1.6-7.1)\end{array}$ & $\begin{array}{c}25.2 \\
(19.8-31.7)\end{array}$ \\
\hline $2016, N=166$ & $\begin{array}{c}68.1 \\
(60.6-74.7)\end{array}$ & $\begin{array}{c}71.7 \\
(64.4-78.0)\end{array}$ & $\begin{array}{c}0.2 \\
(0-1.3)\end{array}$ & $\begin{array}{c}3.6 \\
(1.5-7.8)\end{array}$ & $\begin{array}{c}50.6 \\
(43.1-58.1)\end{array}$ & $\begin{array}{c}0 \\
(0-2.7)\end{array}$ & $\begin{array}{c}27.7 \\
(21.5-35.0)\end{array}$ \\
\hline
\end{tabular}


Table 2. Cont.

\begin{tabular}{|c|c|c|c|c|c|c|c|}
\hline $\begin{array}{c}\text { Species, Origin } \\
\text { Year } \\
\text { No. of Isolates }\end{array}$ & Nalidixic Acid & Ciprofloxacin & Gentamicin & Streptomycin & Tetracycline & Erythromycin & Fully Susceptible \\
\hline \multicolumn{8}{|c|}{ C. jejuni, Turkey } \\
\hline $2010, N=56$ & $\begin{array}{c}60.7 \\
(47.6-72.4)\end{array}$ & $\begin{array}{c}71.4 \\
(58.4-81.7)\end{array}$ & $\begin{array}{c}0 \\
(0-5.8)\end{array}$ & $\begin{array}{c}1.8 \\
(0-10.3)\end{array}$ & $\begin{array}{c}64.3 \\
(51.2-75.6)\end{array}$ & $\begin{array}{c}3.6 \\
(0.3-12.8)\end{array}$ & $\begin{array}{c}19.6 \\
(11.2-32.1)\end{array}$ \\
\hline $2012, N=75$ & $\begin{array}{c}52.0 \\
(40.9-62.9)\end{array}$ & $\begin{array}{c}68.0 \\
(56.8-77.5)\end{array}$ & $\begin{array}{c}0 \\
(0-2.1)\end{array}$ & $\begin{array}{c}9.3 \\
(4.3-18.3)\end{array}$ & $\begin{array}{c}68.0 \\
(56.8-77.5)\end{array}$ & $\begin{array}{c}0 \\
(0-5.8)\end{array}$ & $\begin{array}{c}20.0 \\
(12.5-30.6)\end{array}$ \\
\hline $2014, N=214$ & $\begin{array}{c}57.0 \\
(50.3-63.5)\end{array}$ & $\begin{array}{c}63.1 \\
(56.4-69.3)\end{array}$ & $\begin{array}{c}0 \\
(0-2.3)\end{array}$ & $\begin{array}{c}3.3 \\
(1.5-6.7)\end{array}$ & $\begin{array}{c}56.1 \\
(49.4-62.6)\end{array}$ & $\begin{array}{c}1.9 \\
(0.6-4.9)\end{array}$ & $\begin{array}{c}26.2 \\
(20.7-32.5)\end{array}$ \\
\hline $2016, N=201$ & $\begin{array}{c}73.1 \\
(66.6-78.8)\end{array}$ & $\begin{array}{c}77.6 \\
(71.3-82.8)\end{array}$ & $\begin{array}{c}0 \\
(0-0.8)\end{array}$ & $\begin{array}{c}3.5 \\
(1.6-7.1)\end{array}$ & $\begin{array}{c}52.2 \\
(45.4-59.0)\end{array}$ & $\begin{array}{c}0 \\
(0-2.3)\end{array}$ & $\begin{array}{c}18.4 \\
(13.7-24.4)\end{array}$ \\
\hline
\end{tabular}


Table 3. Association of full susceptibility and resistance to five different antimicrobials of Campylobacter spp. with bacterial species, animal species, and year. Data from $2010-2016$.

\begin{tabular}{|c|c|c|c|c|c|c|c|c|}
\hline Antimicrobial & Covariate & $\begin{array}{l}\text { Coefficient of } \\
\text { Regression }\end{array}$ & Standard Error & Wald & $\mathrm{df}^{1}$ & $p$-Value & Odds Ratio & $\begin{array}{c}95 \% \text { Confidence } \\
\text { Interval of Odds Ratio }\end{array}$ \\
\hline \multirow{3}{*}{$\begin{array}{c}\text { Full } \\
\text { Suscep-tibility }\end{array}$} & Year & 0.006 & 0.039 & 0.025 & 1 & 0.875 & 1.006 & 0.93-1.09 \\
\hline & C. coli vs. C. jejuni & -2.149 & 0.215 & 100.100 & 1 & $<0.001$ & 0.117 & $0.08-1.18$ \\
\hline & Constant & -13.680 & 78.706 & 0.030 & 1 & 0.862 & 0.000 & \\
\hline \multirow{4}{*}{ STR } & Year & -0.01 & 0.05 & 0.01 & 1 & 0.915 & 0.99 & $0.9-1.1$ \\
\hline & C. coli vs. C. jejuni & 1.54 & 0.21 & 53.20 & 1 & $<0.001$ & 4.68 & $3.09-7.09$ \\
\hline & Broilers vs. turkeys & -0.26 & 0.21 & 1.57 & 1 & 0.211 & 0.77 & $0.51-1.16$ \\
\hline & Constant & 7.29 & 98.89 & 0.01 & 1 & 0.941 & $1.47 \times 10^{3}$ & \\
\hline \multirow{4}{*}{ NAL } & Year & 0.08 & 0.03 & 7.67 & 1 & 0.006 & 1.09 & $1.03-1.16$ \\
\hline & C. coli vs. C. jejuni & 1.42 & 0.13 & 125.54 & 1 & $<0.001$ & 4.16 & $3.24-5.33$ \\
\hline & Broilers vs. turkeys & -0.27 & 0.11 & 5.47 & 1 & 0.019 & 0.77 & $0.61-0.96$ \\
\hline & Constant & -169.74 & 61.49 & 7.62 & 1 & 0.006 & 0.00 & \\
\hline \multirow{4}{*}{ CIP } & Year & 0.07 & 0.03 & 4.99 & 1 & 0.025 & 1.08 & $1.01-1.15$ \\
\hline & C. coli vs. C. jejuni & 1.53 & 0.15 & 109.56 & 1 & $<0.001$ & 4.60 & $3.46-6.12$ \\
\hline & Broilers vs. turkeys & -0.32 & 0.12 & 6.90 & 1 & 0.009 & 0.73 & $0.57-0.92$ \\
\hline & Constant & -147.48 & 66.41 & 4.93 & 1 & 0.026 & 0.00 & \\
\hline \multirow{3}{*}{ TET } & C. coli vs. C. jejuni & 1.55 & 0.12 & 163.00 & 1 & $<0.001$ & 4.73 & $3.73-6.01$ \\
\hline & Broilers vs. turkeys & -0.33 & 0.11 & 8.97 & 1 & 0.003 & 0.72 & $0.58-0.89$ \\
\hline & Constant & 165.45 & 61.81 & 7.16 & 1 & 0.007 & $7.1 \times 10^{71}$ & \\
\hline \multirow{4}{*}{ ERY } & Year & -0.33 & 0.04 & 60.89 & 1 & $<0.001$ & 0.72 & $0.66-0.78$ \\
\hline & C. coli vs. C. jejuni & 3.27 & 0.28 & 140.01 & 1 & $<0.001$ & 26.23 & $15.27-45.05$ \\
\hline & Broilers vs. turkeys & -0.53 & 0.19 & 7.62 & 1 & 0.006 & 0.59 & $0.41-0.86$ \\
\hline & Constant & 659.84 & 85.04 & 60.20 & 1 & $<0.001$ & $3.6 \times 10^{286}$ & \\
\hline
\end{tabular}

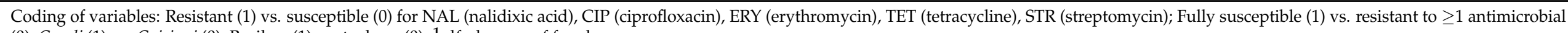
(0); C. coli (1) vs. C. jejuni (0); Broilers (1) vs. turkeys (0). ${ }^{1} \mathrm{df}$ : degrees of freedom. 


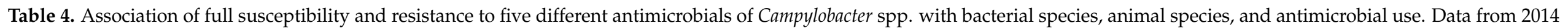
and 2016.

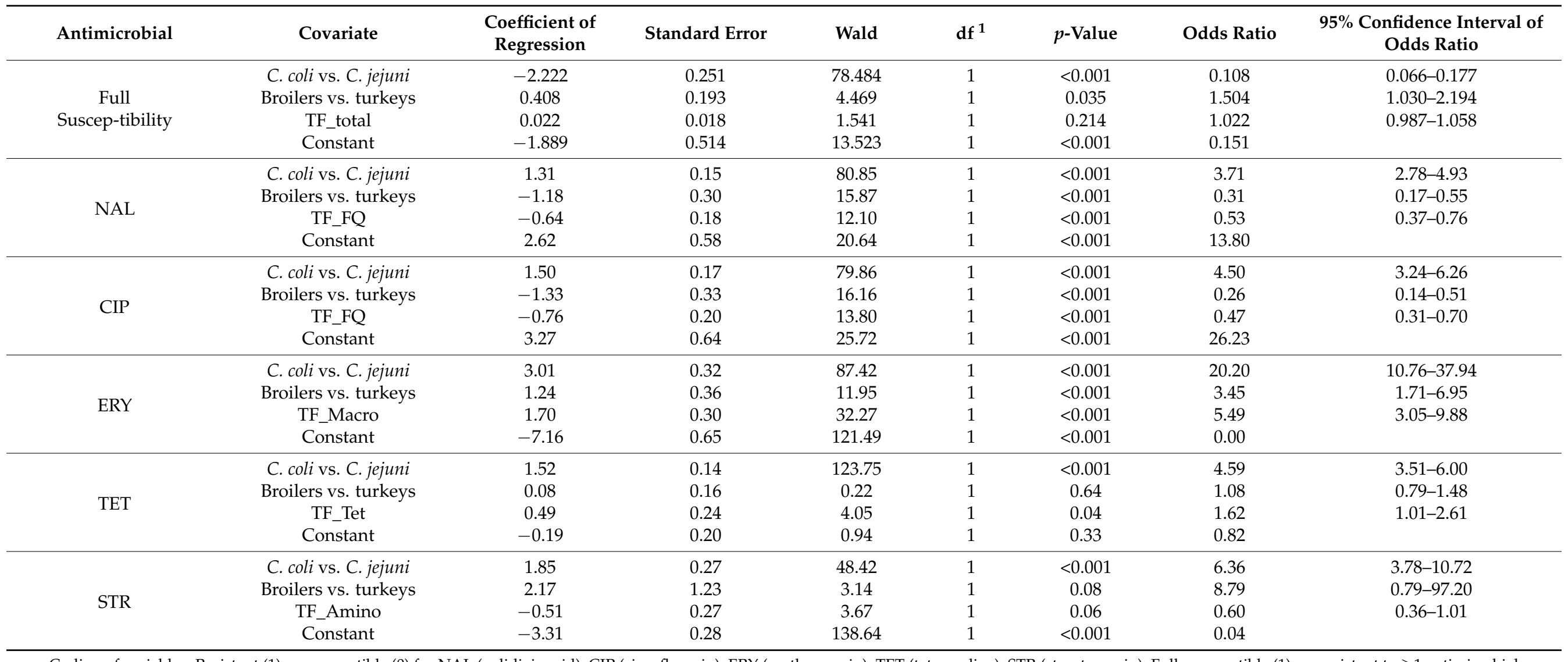

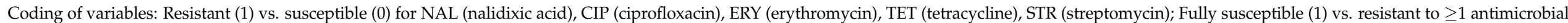

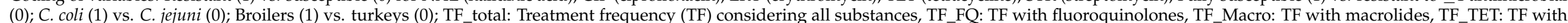
tetracyclines, TF_Amino: TF with aminoglycosides. ${ }^{1} \mathrm{df}$ : degrees of freedom. 
Resistance to each of the antimicrobial substances was more likely to be observed in isolates of $C$. coli than in $C$. jejuni, with odds ratios ranging between 4.2 for nalidixic acid and 26.2 for erythromycin. Resistance to fluoroquinolones increased over the years, while resistance to erythromycin and tetracycline decreased. Resistance to streptomycin did not change significantly in the full model.

Differences between the two animal species were observed for the proportion of fully susceptible isolates and for all substances but aminoglycosides. Resistance was higher in turkeys than in broilers. For both aminoglycosides, the difference was not significant (Table 3).

Stratified analysis considering the two animal species separately confirmed the unchanged proportion of fully susceptible strains for both animal species over time. It was likewise confirmed for both bacterial species when they were considered separately (Tables S1 and S2).

Resistance to streptomycin did not show a significant trend over the years in either animal or bacterial species.

Resistance to nalidixic acid increased significantly in C. jejuni in both animal species between 2010/2011 and 2016, but not in C. coli. The increase in resistance to ciprofloxacin was not significant in both bacterial and animal species over this time period when they were considered separately. Resistance levels to (fluoro-)quinolones of $C$. jejuni ranged between $42.5 \%$ and $77.6 \%$ and in C. coli between $79.5 \%$ and $95.3 \%$ in the individual monitoring years (Table 2).

Resistance to tetracycline decreased in isolates of both Campylobacter spp. from turkeys, but showed no significant trend in isolates from broilers (Tables S1 and S2).

Resistance to erythromycin decreased substantially in C. coli from 2014 to 2016, while resistance in C. jejuni was very low during the whole observation time (15/1014 isolates) (Table 2). Likewise, when isolates from both animal species were considered separately, changes in resistance of $C$. jejuni to erythromycin were not significant, but those in C. coli were in both animal species.

\subsection{Association of $A M R$ and $A M U$}

The association of AMU of specific antimicrobial classes with resistance to substances from these classes could be studied on the basis of data from the years 2014 and 2016 (Tables 2 and 4). There was no significant association of AMU (measured as total AMU) with the proportion of fully susceptible isolates. Resistance to streptomycin was not significantly associated with TF for aminoglycosides in any of the bacteria/animal species combinations.

However, resistance to nalidixic acid in C. jejuni was negatively associated with use of fluoroquinolones measured as TF. The same applied for resistance in C. jejuni to ciprofloxacin in turkeys, but not in broilers (Table S3). No such association was seen for C. coli (Table S4).

Resistance of $C$. coli to tetracycline, on the other hand, was positively associated with use of tetracyclines in turkeys but not in broilers, and no such association was observed for C. jejuni (Table S4). Finally, resistance of C. coli of both animal species to erythromycin was positively associated with the use of macrolides. Resistance of $C$. jejuni to erythromycin was so rare that no significant associations could be observed with logistic regression.

\section{Discussion}

The objective of this study was to analyze trends in AMR in Campylobacter isolates from broilers and turkeys and to study potential associations with AMU in both animal species.

Antimicrobial sales to veterinarians have been decreasing in Germany since 2011 [12,15]. This is in line with decreased sales of antimicrobials for use in animals in a number of other countries [16]. As previously explained in a government report, it could be shown that AMU has decreased in broilers and turkeys between 2014 and 2016 [17]. These detailed data can be considered the best available data on AMU in farm animals in Germany. However, as they were only available for analysis for the interval between 2014 and 2017, and resistance 
data on isolates from poultry according to CID 2013/652/EU are only collected in even years, the analysis had to be restricted to data from the years 2014 to 2016 . We chose to assume that the data of the first half of 2014 were similar to the second half, which is a potential source of bias. It cannot be excluded that AMU was even higher in the first half of the year, i.e., before the implementation of the new legislation. Therefore, the percent change in AMU between the two years may be not accurate. However, only for the use of aminoglycosides in turkeys, this might have affected the direction of change from a slight increase to a slight decrease. Given the minor changes in aminoglycoside use in turkeys, we considered this bias tolerable.

The decrease was observed for most of the considered antimicrobial classes (Table 1). However, it was observed at different levels, and for aminoglycosides there was even a slight increase in TF in turkeys between 2014 and 2016. Moreover, the observed decrease was not always linear. From the data, it is clear that the patterns of AMU also differed between broilers and turkeys, with aminoglycosides being the most frequently used of the investigated substance classes in broilers and fluoroquinolones being most frequently used in turkeys. While the total level of use changed between 2014 and 2016, the share of the different antimicrobial groups considered here did not change substantially. This share differs from data in other countries such as the Netherlands, where use of fluoroquinolones has substantially dropped relative to other classes [18]. It has been previously pointed out that preferences for antimicrobials prescribed for broilers differ substantially between countries [19].

The level of AMU was higher in turkeys than in broilers in the considered time interval, which is in line with reports from other countries $[18,20]$. That applies for the total use, but also for most substance classes that are relevant to Campylobacter, although AMU in turkeys was most frequent for drugs that do not have a direct selection effect on Campylobacter or where resistance was not studied in the monitoring (i.e., penicillins and polymyxins, data not shown).

In particular, TF with quinolones, macrolides, or tetracyclines decreased in turkeys during the indicated time period to similar levels reported for broilers in 2014 at the start of the AMU observation period. An exception was the use of aminoglycosides that was far more frequent in broilers. This was mainly due to a high use of the combination of lincomycin with the aminoglycoside spectinomycin [13]. Differences in AMU patterns between the two animal species indicate that it is not advisable to consider them together as "poultry", which is frequently done in the absence of stratified data [21].

Antimicrobial resistance was higher in C. coli than in C. jejuni, with the exception of resistance to gentamicin, which was rarely seen in both bacterial species originating from both animal species. This finding is in line with previous studies [10], with data collected in the European monitoring systems from food producing animals and humans [6] and with international data [22]. However, some studies also found higher resistance to tetracycline for isolates of $C$. jejuni from broilers as compared to C. coli [23].

Resistance was also higher in isolates from turkeys than in isolates from broilers, with the exception of resistance to the aminoglycosides streptomycin and gentamicin, which did not differ between the isolates from the two animal species. The differences between broilers and turkeys are in line with the pattern of higher levels of use in turkeys for most relevant substances. Given the differences in use of aminoglycosides, a higher resistance in broilers to gentamicin and streptomycin could be expected. Overall, differences in resistance between broilers and turkeys are not pronounced in Europe [6].

In both bacterial and animal species, resistance was highest to fluoroquinolones and tetracycline, which is in line with data from other European countries for the time period [24]. In turkeys, this is consistent with fluoroquinolones and tetracyclines being the most frequently used antimicrobials among the antimicrobials considered in this study (Table 1). In broilers, however, use of tetracycline was low and use of aminoglycosides was substantially higher than that of fluoroquinolones (Table 1). 
The AMR characteristics in the two bacterial species isolated from the same animal species were different during the whole observation period. Resistance to nalidixic acid increased in C. jejuni from turkeys and broilers, but not in C. coli. However, it has to be noted that resistance of $C$. coli to fluoroquinolones was already above $87 \%$ in both animal species in the first year of observation (2010 for turkeys and 2011 for broilers), making a further increase unlikely to be statistically significant. The proportion of $C$. coli isolates from turkeys that was resistant to nalidixic acid was $\geq 87 \%$ at any time between 2011 and 2016. In contrast, C. jejuni exhibited resistance rates to nalidixic acid $<75 \%$ throughout the observation period. Resistance to ciprofloxacin did not increase significantly in either animal or bacterial species when considered separately (Table 2). Resistance rates to fluoroquinolones in C. jejuni from both species were between $58 \%$ and $71 \%$ in 2010 and 2011. A numerical increase in resistance to ciprofloxacin and nalidixic acid was observed in C. jejuni between 2014 and 2016 despite decreasing use of fluoroquinolones in that period. This is, on first sight, counterintuitive. A possible reason is that the level of use in 2015 and 2016 was, despite the decreasing overall trend, still high enough to support resistance selection in the population. However, so far, the use level of fluoroquinolones that would not continue selecting resistant $C$. jejuni remains to be determined. Data from the Netherlands indicate that resistance of $C$. jejuni from broilers to ciprofloxacin may even increase when the amount of use of fluoroquinolones in the population is very low [24,25]. Increases in resistance of Campylobacter spp. to fluoroquinolones have also been observed by others that likewise did not report a parallel increase in fluoroquinolone use [26]. It has been demonstrated that resistance to fluoroquinolones is associated with other selective advantages of distinct C. jejuni strains for colonization of broilers [27], leading to an increase in resistance despite reduced use. Furthermore, clonal spreading of (fluoro)quinolone-resistant C. jejuni was observed in European countries [28,29]. However, these associations are embedded in complex relationships that require further study [30]. On the other hand, on-farm use of fluoroquinolones was shown to be the major risk factor for ciprofloxacin resistant Campylobacter spp. on broiler farms [8]. Increases in resistance to fluoroquinolones in Campylobacter spp. are of concern, as resistance in Campylobacter from animals has been shown to be associated with resistance of Campylobacter from human infections [3]. Moreover, fluoroquinolones are among WHOs "Highest Priority Critically Important Antimicrobials" (HPCIA) [31].

Resistance to the aminoglycoside streptomycin remained unchanged in both Campylobacter spp. from both animal species. Streptomycin resistance was observed in both bacterial and animal species in all years. However, it did not differ between the animal species despite substantial differences in aminoglycoside use in broilers and turkeys (Table 1). The proportion of resistant isolates is in line with reports from the US for poultry meat [32] and within the range of reported resistance in Campylobacter in other EU member states [6] and international data [22]. Aminoglycoside use did only decrease temporarily in broilers, and it even increased slightly in turkeys, which is in line with unchanged resistance rates.

Resistance to gentamicin, the other aminoglycoside included in the testing, was close to zero in both animal and bacterial species at all times, with only one isolate of C. jejuni from broilers and one isolate of $C$. coli from turkeys being resistant. Low resistance in Campylobacter spp. to gentamicin is consistent with reports from other EU member states [6] and Canada $[23,26]$. Resistance in humans in Europe tends to be higher but is still in the range of $0-1.5 \%$ for both Campylobacter spp. [6].

The decrease of resistance to erythromycin in C. coli from turkeys and broilers over the whole observation period is welcome from a public health perspective, as macrolides are considered HPCIA by WHO [31]. In C. jejuni, resistance to erythromycin was rare in broilers and turkeys with only 15 of 1014 resistant isolates $(1.5 \%)$, which is in agreement with data on resistance of C. jejuni from humans and poultry in Europe [6] and Campylobacter from broilers in other countries [23]. Resistance to erythromycin in C. coli was positively associated with TF for macrolides, i.e., a reduction in macrolide use was associated with a 
decrease in AMR in C. coli. Resistance to macrolides is mainly conferred by a point mutation in the 23S rRNA gene [33]. It was observed that C. jejuni strains lose fitness upon acquisition of the point-mutation-based resistance determinant but that this phenomenon does not apply for C. coli [34], eventually explaining the observed difference in macrolide resistance prevalence in both species observed in this study. Recently, circulation of an erm $\mathrm{B}$ resistance cassette was observed in China and Spain, but ermB in Campylobacter spp. is still rare in Europe $[35,36]$. Since this resistance mechanism does not seem to interfere with fitness of C. jejuni, further reduction of AMU of macrolides in poultry is highly recommended in order to avoid the risk of selection and spread of novel ermB carrying clonal lineages.

In isolates from turkeys, a substantial decrease in AMR was observed to tetracycline in both C. jejuni and C. coli over the whole observation period (2010 to 2016). This is in contrast to data from other countries such as Canada, where increasing resistance to tetracycline was observed in Campylobacter from turkeys [26], although there was substantial variation between years. In broilers, resistance of the two bacterial species to tetracycline did not change significantly over time. A reduction of the use of tetracyclines was observed in both animal species between 2014 and 2016, but an associated decrease in AMR was only seen in C. coli from turkeys, despite the fact that use tended to be higher in turkeys than in broilers and that the reduction in use was more pronounced in broilers $(-62 \%)$ than in turkeys $(-38 \%)$.

The differences in trend between the bacterial species indicate that it is not AMU alone that drives differences in resistance, as both bacterial species are derived from the same overall population and therefore exposed to similar levels of antimicrobial treatment over time. These differences between the bacterial species have also been observed when comparing AMR in Campylobacter from organic and conventional turkey meat [10] and in European trend reports [6,24]. As pointed out above, for some antimicrobials, this can be attributed to differences in fitness costs associated with resistance. However, further research is needed to fully understand these differences.

\section{Materials and Methods}

\subsection{Data on AMR of Campylobacter spp. in Broilers and Turkeys}

Antimicrobial resistance data on Campylobacter were taken from investigations carried out in the framework of a national monitoring program that is conducted under Directive 2003/99/EC and in compliance with Commission Implementing Decision (CID) 2013/652/EU. The monitoring has been in place since 2009 and is implemented following a specific national legal framework [37]. Before 2014, sampling and testing were carried out in a very similar way to that later prescribed by the CID. Aggregated data are routinely reported in national reports [38-43]. Caeca from broilers were included in the monitoring in 2011, 2013, 2014, and 2016. Caeca from turkeys were included in 2010, 2012, 2014, and 2016. Caeca were collected at slaughter. Samples were collected across Germany according to the regional slaughterhouse throughput of the respective animal species. Regional slaughterhouse throughput was obtained from the Federal Office of Statistics (www.destatis.de). One pooled sample, composed of the content of 10 caeca from birds from the same randomly chosen slaughter batch, was tested for Campylobacter, and a maximum of one isolate per Campylobacter species was submitted for further testing. Samples were tested in the regional laboratories of the federal states according to ISO 10272-1:2017, procedure B, and $1 \mathrm{~g}$ of the caecal material was used as test portion. In brief, the samples were 1:10 (w/v) diluted in Preston broth and incubated for $24 \pm 2 \mathrm{~h}$ at $41.5 \pm 1{ }^{\circ} \mathrm{C}$ under microaerobic atmosphere. After this enrichment step, a loop of $10 \mu \mathrm{L}$ was spread on modified charcoal cefoperazone deoxycholate agar (mCCDA), and incubation was performed for another $44 \pm 4 \mathrm{~h}$ under the same conditions. At least one typical or suspect Campylobacter colony was confirmed using either phenotypic tests as described in ISO 10272-1:2017 or MALDI-TOF or PCR methods, which were in-house validated in the respective regional laboratories. According to ISO 10272-1:2017, if the first colony was negative, up to four more suspect colonies were checked unless the sample was considered negative for thermophilic Campylobacter spp. 
Per sample, one or two representative isolates of Campylobacter spp. were speciated by real-time PCR [44], and one or two (in case both C. jejuni and C. coli were isolated from the same sample) isolates per sample were tested for AMR according to the prescriptions given in CID 2013/652/EU [5]. For this purpose, strains were subcultured on Columbia blood agar for $24 \pm 2 \mathrm{~h}$ at $41.5 \pm 1{ }^{\circ} \mathrm{C}$ under microaerobic atmosphere $\left(5 \% \mathrm{O}_{2}, 10 \% \mathrm{CO}_{2}\right.$, rest $\mathrm{N}_{2}$ ). Broth microdilution susceptibility testing was performed according to M45-A [45] and VET06 [46] with the in-house validated modification of use of fetal calf serum instead of lysed horse blood in the culture medium for improved readability of Campylobacter growth. Cation-supplemented Mueller-Hinton broth (MSC Diagnostics, Swalmen, The Netherlands) supplemented with $5 \%$ fetal calf serum (PAN Biotech, Aidenbach, Germany) was inoculated with $2-8 \times 10^{5}$ colony-forming units $/ \mathrm{mL}$ using bacteria grown on Columbia blood agar. Minimum inhibitory concentrations (MICs) were determined for six antimicrobials (Table 2) using the European standardized microtiter plate formats EUCAMP (2010-2013), and EUCAMP2 (2014-2016) (TREK Diagnostic Systems, Altrincham, United Kingdom). Incubation was performed for $44 \pm 4 \mathrm{~h}$ at $37^{\circ} \mathrm{C}$ under microaerobic atmosphere. MICs (mg/L) were semi-automatically analyzed using the Sensititre Vizion system (Thermo Fisher Scientific, Waltham, MA, USA) and the SWIN-Software (Thermo Fisher Scientific, Waltham, MA, USA). MIC were evaluated according to the epidemiological cutoff values (ECOFF) published by the European Committee for Antimicrobial Susceptibility Testing (EUCAST; https: / / mic.eucast.org/Eucast2 accessed 1 July 2020) and laid down in the CID 2013/652/EU.

\subsection{Data on AMU in Broilers and Turkeys}

Antimicrobial sales data have been collected in Germany since 2011, and data showed a decrease in overall sales from the very beginning [12]. However, the sales data do not allow for an estimation of the use of antimicrobials in specific animal species [3,21].

Specific data on AMU in broilers and turkeys in Germany have been available since July 2014 in the framework of the national data collection on AMU in animals for meat production according to Section $58 \mathrm{a}-\mathrm{c}$ of the German Medicinal Products Act [47]. In this section of the German Medicinal Products Act, the national minimization strategy for AMU in animals is defined. This strategy is based on obligatory data reporting and a benchmarking process where AMU in farms is compared to the median and the 3 rd quartile of AMU in all farms of the same field of production [17]. The key indicator calculated is a TF per animal per half-year. To this end, farmers are required to report each treatment of their animals with antimicrobials, and data are collected in a central database. Twice a year, i.e., for each half-year period, the database calculates the TF for each farm using the following formula:

$$
\mathrm{TF}=\frac{\sum_{i} n(i) \times d(i) \times s(i)}{\overline{\mathrm{N}}}
$$

where $n(i), d(i)$, and $s(i)$ are the number of animals treated, the treatment duration in days, and the number of active antimicrobial substances, respectively, in the $i$ th treatment that was administered on the farm in the half-year period, and where $\overline{\mathrm{N}}$ is the average number of animals housed on the farm during that period. In essence, the formula divides the total number of animal days under treatment in the half-year period by the average number of animals housed on the farm during the respective period. To this end, every antimicrobial substance is being counted on its own, i.e., if animals receive two antimicrobials on a given day, this is considered as two treatment days.

While not part of the official minimization strategy, treatment frequencies can also be calculated for specific classes of antimicrobials:

$$
\mathrm{TF}_{\mathcal{c}}=\frac{\sum_{i} n(i) \times d(i) \times s_{\mathcal{c}}(i)}{\overline{\mathrm{N}}}
$$

where $s_{c}(i)$ is the number of active substances belonging to class $c$ in the $i$ th treatment. The sum of all class-specific treatment frequencies retrieves the total TF given by formula 
(1). While the minimization strategy with its benchmarking process targets farm level AMU, for the present study, we used national level treatment frequencies, i.e., the sums in formulas (1) and (2) are taken over all treatments on all farms in the respective field of production, and the denominator is the sum of all average numbers of animals housed on all farms.

These use data were taken from a supplement to the national report on the evaluation of the legal provisions that was published by the German government in 2019 [13]. Use data had been extracted from the national database and pseudonymized in spring 2018 in the framework of the evaluation process. Data were submitted to extensive plausibility checks to exclude erroneous entries to the best extent possible. Details of these plausibility checks have been reported in a government report [13]. They considered, among others, discrepancies between numbers of animal days under treatment and amount of drug used and implausible animal numbers, indicating erroneous entries. Identified erroneous entries were excluded from the analyses. For our study, we used the total as well as the antimicrobial class-specific national level treatment frequencies provided in the government report [13].

\subsection{Statistical Analyses}

AMR data: For the analysis, MICs from the included isolates were categorized into susceptible and resistant, as prescribed by CID 2013/652/EU, using the epidemiological cut-off values provided by EUCAST and included in the CID. Proportions of resistant isolates are presented as crude percentages with 95\% confidence intervals [14]. Two sets of logistic regression analyses were run per antimicrobial using the statistical analysis software SPSS (IBM Deutschland GmbH, 71139 Ehningen Germany). In both analyses the dependent variable was resistant (1) vs. susceptible (0) to the drug in question. In addition to the individual drugs, an outcome variable "full susceptibility" was defined as an isolate susceptible to all tested antimicrobials. It was coded as "fully susceptible (1)" and "not fully susceptible (0)", i.e., resistant to at least one of the substances tested. This full susceptibility (to a predefined set of substances) parameter has recently been recommended as a proper indicator of AMR in animals [48].

One set of analyses included data from the whole time period, i.e., 2010 to 2016 in order to identify the overall trend in resistance in Campylobacter from poultry. Besides the numerical variable year, animal species (broilers $=1$, turkeys $=0$ ) and bacterial species (C. coli $=1$, C. jejuni $=0$ ) were included as independent variables.

In a second set of regressions, the outcome variables were identical. However, here the variable "year" was replaced by a variable "use" using the numerical value of the TF for the antimicrobial class that the test substance belonged to in the respective year, i.e., aminoglycosides for streptomycin, macrolides for erythromycin, fluoroquinolones for ciprofloxacin and nalidixic acid, and tetracyclines for tetracycline. For the analysis of full susceptibility, the total TF over all antimicrobials including those that were not considered in the substance specific analysis for the respective year was the use variable.

As specific use data have only been available since July 2014, the association with AMU was only studied using resistance data from 2014 and 2016. Use data in the first half of 2014 were assumed to be similar to the second half of that year. For 2016, the mean between the two TF values for the 6 month periods was calculated and used in the analysis. The change in use of substance classes over time was calculated as the difference of the mean of TF in 2016 and the TF in the second half of 2014 (2014/2) divided by the TF in 2014/2, expressed as a percentage.

Further sub-analyses were run in addition to the two full models to better characterize the observed differences (Supplementary Tables S1-S4). To this end, separate regressions were run for $C$. jejuni and C. coli as differences in AMR between the two bacterial species are well established $[6,10]$. Likewise, separate regressions were run for the two animal species, as from the use data it became clear that use data differ between the species and likewise AMR differed between animal species in the full model. Finally, separate regressions were 
run for all combinations of animal and bacterial species. For all analyses, $p$-values $<0.05$ were considered significant.

\section{Conclusions}

The association of AMU and AMR differed between the two Campylobacter spp. and the six antimicrobials considered. Overall, AMU decreased, but this was not associated with a decrease in AMR for all combinations of antimicrobials and bacterial species. Resistance to tetracycline and erythromycin tended to decrease when use decreased. For fluoroquinolones, an opposite trend was observed, which is consistent with the resistance mechanism possibly conferring advantages in distinct Campylobacter lineages. This underlines the importance of having detailed use data on the national level. It warrants on the one hand further investigations over longer time periods and on the other hand molecular investigations for a more in-depth understanding of the underlying mechanisms.

Supplementary Materials: The following are available online at https:/ /www.mdpi.com/article/10 $.3390 /$ antibiotics10060673/s1. Table S1. Association of year with full susceptibility and resistance to antimicrobials in C. jejuni from broilers and turkeys. Table S2. Association of year with full susceptibility and resistance to antimicrobials in C. coli from broilers and turkeys. Table S3. Association of therapy frequency with full susceptibility and resistance to antimicrobials in C. jejuni from broilers and turkeys. Table S4. Association of therapy frequency with full susceptibility and resistance to antimicrobials in C. coli from broilers and turkeys. Table S5: Minimum inhibitory concentrations of isolates included in the analysis.

Author Contributions: Conceptualization, B.-A.T.; data curation, B.-A.T., M.F., K.A. and K.S.; formal analysis, B.-A.T.; investigation, M.-T.K., C.B., M.F., A.K. and K.S.; writing-original draft, B.-A.T.; writing-review and editing, B.-A.T., M.F., K.A., A.K. and K.S. All authors have read and agreed to the published version of the manuscript.

Funding: This research received no external funding.

Institutional Review Board Statement: Not applicable.

Informed Consent Statement: Not applicable.

Data Availability Statement: Data on AMR are available in the supplementary tables. Data on use are available at: https://www.bmel.de/DE/themen/tiere/tierarzneimittel/kurzfassung16-amgnovelle.html.

Acknowledgments: The authors gratefully acknowledge the support of the regional state laboratories that provided the isolates included in this manuscript. Without their support this research would not have been possible.

Conflicts of Interest: The authors declare no conflict of interest.

\section{References}

1. EFSA; ECDC. The European Union One Health 2019 Zoonoses Report. EFSA J. 2021, 19, 6406. [CrossRef]

2. Rosner, B.M.; Schielke, A.; Didelot, X.; Kops, F.; Breidenbach, J.; Willrich, N.; Golz, G.; Alter, T.; Stingl, K.; Josenhans, C.; et al. A combined case-control and molecular source attribution study of human Campylobacter infections in Germany, 2011-2014. Sci. Rep. 2017, 7, 5139. [CrossRef]

3. ECDC; EFSA; EMA. ECDC/EFSA/EMA second joint report on the integrated analysis of the consumption of antimicrobial agents and occurrence of antimicrobial resistance in bacteria from humans and food-producing animals. Joint Interagency Antimicrobial Consumption and Resistance Analysis (JIACRA) Report. EFSA J. 2017, 15, 4872. [CrossRef]

4. Mughini-Gras, L.; Penny, C.; Ragimbeau, C.; Schets, F.M.; Blaak, H.; Duim, B.; Wagenaar, J.A.; de Boer, A.; Cauchie, H.M.; Mossong, J.; et al. Quantifying potential sources of surface water contamination with Campylobacter jejuni and Campylobacter coli. Water Res. 2016, 101, 36-45. [CrossRef]

5. European Commission. Commission Implementing Decision of 12 November 2013 on the monitoring and reporting of antimicrobial resistance in zoonotic and commensal bacteria, 2013/652/EU. Off. J. Eur. Union 2013, L303, 26-39.

6. EFSA; ECDC. The European Union summary report on antimicrobial resistance in zoonotic and indicator bacteria from humans, animals and food in 2017/2018. EFSA J. 2020, 18, 6007. [CrossRef] 
7. $\quad$ Luiken, R.E.C.; Van Gompel, L.; Munk, P.; Sarrazin, S.; Joosten, P.; Dorado-García, A.; Borup Hansen, R.; Knudsen, B.E.; Bossers, A.; Wagenaar, J.A.; et al. Associations between antimicrobial use and the faecal resistome on broiler farms from nine European countries. J. Antimicrob. Chemother. 2019, 74, 2596-2604. [CrossRef]

8. Taylor, N.M.; Wales, A.D.; Ridley, A.M.; Davies, R.H. Farm level risk factors for fluoroquinolone resistance in E. coli and thermophilic Campylobacter spp. on poultry farms. Avian Pathol. 2016, 45, 559-568. [CrossRef]

9. Dorado-Garcia, A.; Mevius, D.J.; Jacobs, J.J.; Van Geijlswijk, I.M.; Mouton, J.W.; Wagenaar, J.A.; Heederik, D.J. Quantitative assessment of antimicrobial resistance in livestock during the course of a nationwide antimicrobial use reduction in the Netherlands. J. Antimicrob. Chemother. 2016, 71, 3607-3619. [CrossRef] [PubMed]

10. Tenhagen, B.A.; Alt, K.; Kasbohrer, A.; Kollas, C.; Pfefferkorn, B.; Naumann, S.; Wiehle, L.; Thieck, M.; Stingl, K. Comparison of Antimicrobial Resistance of Thermophilic Campylobacter Isolates from Conventional and Organic Turkey Meat in Germany. Foodborne Pathog. Dis. 2020, 17, 750-757. [CrossRef] [PubMed]

11. Kempf, I.; Kerouanton, A.; Bougeard, S.; Nagard, B.; Rose, V.; Mourand, G.; Osterberg, J.; Denis, M.; Bengtsson, B.O. Campylobacter coli in Organic and Conventional Pig Production in France and Sweden: Prevalence and Antimicrobial Resistance. Front. Microbiol. 2017, 8, 955. [CrossRef]

12. Koper, L.M.; Bode, C.; Bender, A.; Reimer, I.; Heberer, T.; Wallmann, J. Eight years of sales surveillance of antimicrobials for veterinary use in Germany-What are the perceptions? PLOS ONE 2020, 15, e0237459. [CrossRef]

13. Flor, M.; Käsbohrer, A.; Kaspar, H.; Tenhagen, B.-A.; Wallmann, J. Beiträge der Arbeitsgruppe Antibiotikaresistenz zur Evaluierung der 16. AMG-Novelle-Themenkomplex 1: Entwicklung der Antibiotikaabgabe- und-Verbrauchsmengen Sowie der Therapiehäufigkeit; Bundesministerium für Ernährung und Landwirtschaft: Berlin, Germany, 2019.

14. Agresti, A.; Coull, B.A. Approximate is Better than "Exact" for Interval Estimation of Binomial Proportions. Am. Stat. 1998, 52, 119-126. [CrossRef]

15. Wallmann, J.; Bender, A.; Bode, C.; Köper, L.M.; Heberer, T. Abgabemengen antimikrobiell wirksamer Stoffe in Deutschland 2016. Auswertung der nach DIMDI-AMV eingereichten Daten 2016 und Vergleich mit den Daten aus den Vorjahren. Dtsch. Tierärzteblatt 2017, 65, 1650-1659.

16. EMA. Sales of Veterinary Antimicrobial Agents in 31 European Countries in 2018-Trends from 2010 to 2018. Tenth ESVAC Report; European Medicines Agency (EMA): Amsterdam, The Netherlands, 2020; p. 184.

17. BMEL. Bericht des Bundesministeriums für Ernährung und Landwirtschaft über die Evaluierung des Antibiotikaminimierungskonzepts der 16. AMG-Novelle; Bundesministerium für Ernährung und Landwirtschaft: Berlin, Germany, 2019.

18. SDA. Het Gebruik van Antibiotica bij Landbouwhuisdieren in 2019: Trends, Benchmarken, Bedrijven en Dierenartsen; Autoriteit Diergeneesmiddelen, SDa: Utrecht, The Netherlands, 2020; p. 36.

19. Joosten, P.; Sarrazin, S.; Van Gompel, L.; Luiken, R.E.C.; Mevius, D.J.; Wagenaar, J.A.; Heederik, D.J.J.; Dewulf, J. Quantitative and qualitative analysis of antimicrobial usage at farm and flock level on 181 broiler farms in nine European countries. J. Antimicrob. Chemother. 2019, 74, 798-806. [CrossRef]

20. ITAVI. Réseau Professionnel de Références sur les Usages d'Antibiotiques en Élevage Avicole; ITAVI: Lyon, France, 2019.

21. Mesa Varona, O.; Chaintarli, K.; Muller-Pebody, B.; Anjum, M.F.; Eckmanns, T.; Norstrom, M.; Boone, I.; Tenhagen, B.A. Monitoring Antimicrobial Resistance and Drug Usage in the Human and Livestock Sector and Foodborne Antimicrobial Resistance in Six European Countries. Infect. Drug Resist. 2020, 13, 957-993. [CrossRef]

22. Signorini, M.L.; Rossler, E.; Diaz David, D.C.; Olivero, C.R.; Romero-Scharpen, A.; Soto, L.P.; Astesana, D.M.; Berisvil, A.P.; Zimmermann, J.A.; Fusari, M.L.; et al. Antimicrobial Resistance of Thermotolerant Campylobacter Species Isolated from Humans, Food-Producing Animals, and Products of Animal Origin: A Worldwide Meta-Analysis. Microb. Drug Resist. 2018, 24, 1174-1190. [CrossRef]

23. Varga, C.; Guerin, M.T.; Brash, M.L.; Slavic, D.; Boerlin, P.; Susta, L. Antimicrobial resistance in Campylobacter jejuni and Campylobacter coli isolated from small poultry flocks in Ontario, Canada: A two-year surveillance study. PLoS ONE 2019, 14, e0221429. [CrossRef] [PubMed]

24. EFSA; ECDC. The European Union summary report on antimicrobial resistance in zoonotic and indicator bacteria from humans, animals and food in 2016. EFSA J. 2018, 16, 5182. [CrossRef]

25. SDA. Het Gebruik van Antibiotica bij Landbouwhuisdieren in 2016: Trends, Benchmarken, Bedrijven en Dierenartsen; Autoriteit Diergeneesmiddelen, SDa: Utrecht, The Netherlands, 2017; p. 93.

26. Agunos, A.; Gow, S.P.; Léger, D.F.; Carson, C.A.; Deckert, A.E.; Bosman, A.L.; Loest, D.; Irwin, R.J.; Reid-Smith, R.J. Antimicrobial Use and Antimicrobial Resistance Indicators-Integration of Farm-Level Surveillance Data From Broiler Chickens and Turkeys in British Columbia, Canada. Front. Vet. Sci. 2019, 6, 131. [CrossRef]

27. Luo, N.; Pereira, S.; Sahin, O.; Lin, J.; Huang, S.; Michel, L.; Zhang, Q. Enhanced in vivo fitness of fluoroquinolone-resistant Campylobacter jejuni in the absence of antibiotic selection pressure. Proc. Natl. Acad. Sci. USA 2005, 102, 541-546. [CrossRef]

28. Kovac, J.; Cadez, N.; Stessl, B.; Stingl, K.; Gruntar, I.; Ocepek, M.; Trkov, M.; Wagner, M.; Smole Mozina, S. High genetic similarity of ciprofloxacin-resistant Campylobacter jejuni in central Europe. Front. Microbiol. 2015, 6, 1169. [CrossRef]

29. Technical University of Denmark-National Food Institute. Comparative genomics of quinolone-resistant and susceptible Campylobacter jejuni of poultry origin from major poultry producing European countries (GENCAMP). EFSA Supporting Publ. 2018, EN-1398, 36. [CrossRef] 
30. Zeitouni, S.; Kempf, I. Fitness cost of fluoroquinolone resistance in Campylobacter coli and Campylobacter jejuni. Microb. Drug Resist. 2011, 17, 171-179. [CrossRef]

31. WHO. Critically Important Antimicrobials for Human Medicine, 6th Revision 2018; World Health Organisation: Genf, Switzerland, 2019; p. 52.

32. Noormohamed, A.; Fakhr, M.K. Prevalence and Antimicrobial Susceptibility of Campylobacter spp. in Oklahoma Conventional and Organic Retail Poultry. Open Microbiol. J. 2014, 8, 130-137. [CrossRef]

33. Bolinger, H.; Kathariou, S. The Current State of Macrolide Resistance in Campylobacter spp.: Trends and Impacts of Resistance Mechanisms. Appl. Environ. Microbiol. 2017, 83. [CrossRef]

34. Zeitouni, S.; Collin, O.; Andraud, M.; Ermel, G.; Kempf, I. Fitness of macrolide resistant Campylobacter coli and Campylobacter jejuni. Microb. Drug Resist. 2012, 18, 101-108. [CrossRef]

35. Florez-Cuadrado, D.; Ugarte-Ruiz, M.; Quesada, A.; Palomo, G.; Dominguez, L.; Porrero, M.C. Description of an erm(B)-carrying Campylobacter coli isolate in Europe. J. Antimicrob. Chemother. 2016, 71, 841-843. [CrossRef]

36. Wang, Y.; Zhang, M.; Deng, F.; Shen, Z.; Wu, C.; Zhang, J.; Zhang, Q.; Shen, J. Emergence of multidrug-resistant Campylobacter species isolates with a horizontally acquired rRNA methylase. Antimicrob. Agents Chemother. 2014, 58, 5405-5412. [CrossRef] [PubMed]

37. BMEL. Allgemeine Verwaltungsvorschrift über die Erfassung, Auswertung und Veröffentlichung von Daten über das Auftreten von Zoonosen und Zoonoseerregern entlang der Lebensmittelkette (AVV Zoonosen Lebensmittelkette), Zuletzt geändert durch Verwaltungsvorschrift vom 19. Juni 2017 (BAnz AT 23.06.2017 B2). Bundesanzeiger 2012, Nr.27, 623.

38. BVL. Berichte zur Lebensmittelsicherheit-Zoonosen-Monitoring 2016; Bundesamt für Verbraucherschutz und Lebensmittelsicherheit: Berlin, Germany, 2017; Volume 11, p. 77.

39. BVL. Berichte zur Lebensmittelsicherheit-Zoonosen-Monitoring 2014; Bundesamt für Verbraucherschutz und Lebensmittelsicherheit: Berlin, Germany, 2016; Volume 10, p. 63.

40. BVL. Berichte zur Lebensmittelsicherheit 2013-Zoonosen-Monitoring; Bundesamt für Verbraucherschutz und Lebensmittelsicherheit: Berlin, Germany, 2015; Volume 9, p. 70.

41. BVL. Berichte zur Lebensmittelsicherheit 2012-Zoonosen-Monitoring; Bundesamt für Verbraucherschutz und Lebensmittelsicherheit: Berlin, Germany, 2014; Volume 8, p. 72.

42. BVL. Berichte zur Lebensmittelsicherheit 2011-Zoonosen-Monitoring; Bundesamt für Verbraucherschutz und Lebensmittelsicherheit: Berlin, Germany, 2013; Volume 7, p. 76.

43. BVL. Berichte zur Lebensmittelsicherheit 2010-Zoonosen-Monitoring; Bundesamt für Verbraucherschutz und Lebensmittelsicherheit: Berlin, Germany, 2012; Volume 6, p. 61.

44. Mayr, A.M.; Lick, S.; Bauer, J.; Tharigen, D.; Busch, U.; Huber, I. Rapid detection and differentiation of Campylobacter jejuni, Campylobacter coli, and Campylobacter lari in food, using multiplex real-time PCR. J. Food Prot. 2010, 73, 241-250. [CrossRef] [PubMed]

45. Clinical and Laboratory Standards Institute. M45-A: Methods for Antimicrobial Dilution and Disk Susceptibility Testing of Infrequently Isolated or Fastidious Bacteria; Clinical and Laboratory Standards Institute: Wayne, PA, USA, 2015.

46. Clinical and Laboratory Standards Institute. CLSI VET06: Methods for Antimicrobial Susceptibility Testing of Infrequently Isolated or Fastidious Bacteria Isolated from Animals; Clinical and Laboratory Standards Institute: Wayne, PA, USA, 2017.

47. Anonymous. Arzneimittelgesetz in der Fassung der Bekanntmachung vom 12. Dezember 2005 (BGBl. I S. 3394), das Zuletzt Durch Artikel 1 der Verordnung vom 19. Mai 2021 (BGBl. I S. 1164) Geändert Worden Ist; Bundesregierung: Berlin, Germany, 2021.

48. Cormican, M.; Hopkins, S.; Jarlier, V.; Reilly, J.; Simonsen, G.S.; Strauss, R.; Vandenberg, O.; Zabicka, D.; Zarb, P.; Catchpole, M.; et al. ECDC, EFSA and EMA Joint Scientific Opinion on a list of outcome indicators as regards surveillance of antimicrobial resistance and antimicrobial consumption in humans and food-producing animals. EFSA J. 2017, 15, 70. [CrossRef] 